\title{
UK's NICE updates COVID-19 guideline to include monoclonal antibody
} recommendations

UK's National Institute for Health and Care Excellence (NICE) has updated its Managing COVID-19 rapid guidelines to include recommendations on the use of monoclonal antibodies for patients with COVID-19 in hospital.

The recommendations suggest offering a combination of casirivimab and imdevimab [Ronapreve, REGEN-COV, or REGEN-COV2; Regeneron Pharmaceuticals] to hospitalised COVID-19 patients aged $\geq 12$ years. Patients must be seronegative (no existing levels of SARS-CoV-2 antibodies in their system), otherwise there will be no benefit of the treatment.

"This updated advice will help ensure that COVID-19 patients in hospital have access to the latest treatments, and that healthcare workers are aware which patients will benefit from these treatments", commented Director of NICE's Centre for Guidelines, Dr Paul Chrisp.

National Institute for Health and Care Excellence (NICE). NICE updates managing COVID guideline with new monoclonal antibody recommendations. Internet Document : 4 Oct 2021. Available from: URL: https://www.nice.org.uk/news/article/nice-updates-managing-covid-guideline-with-new-monoclonal-antibody-recommendations 